\title{
Low-Loss Hollow-Core Anti-Resonant Fibers With Semi-Circular Nested Tubes
}

\author{
Habib, Selim; Bang, Ole; Bache, Morten
}

Published in:

IEEE Journal of Selected Topics in Quantum Electronics

Link to article, DOI:

10.1109/JSTQE.2015.2512989

Publication date:

2016

Document Version

Peer reviewed version

Link back to DTU Orbit

Citation $(A P A)$ :

Habib, S., Bang, O., \& Bache, M. (2016). Low-Loss Hollow-Core Anti-Resonant Fibers With Semi-Circular Nested Tubes. IEEE Journal of Selected Topics in Quantum Electronics, 22(2), [4402107].

https://doi.org/10.1109/JSTQE.2015.2512989

\section{General rights}

Copyright and moral rights for the publications made accessible in the public portal are retained by the authors and/or other copyright owners and it is a condition of accessing publications that users recognise and abide by the legal requirements associated with these rights.

- Users may download and print one copy of any publication from the public portal for the purpose of private study or research.

- You may not further distribute the material or use it for any profit-making activity or commercial gain

- You may freely distribute the URL identifying the publication in the public portal

If you believe that this document breaches copyright please contact us providing details, and we will remove access to the work immediately and investigate your claim. 


\title{
Low-loss Hollow-core Anti-Resonant Fibers with Semi-Circular Nested Tubes
}

\author{
Md. Selim Habib, Student Member, IEEE, Ole Bang, and Morten Bache
}

\begin{abstract}
Hollow-core fibers with a single ring of circular anti-resonant tubes as the cladding provide a simple way of getting a negative-curvature hollow-core, resulting in broadband low-loss transmission with little power overlap in the glass. These fibers show a significant improvement in loss performance if the anti-resonant tubes have nested tubes inside them, and here we investigate the role of the shape and position of these nested elements. By allowing the circular nested elements to become semi-circular we selectively change the position or curvature of the nested elements. We find that the loss performance is quite insensitive to the curvature of the nested element, while the distance from the core boundary to the outer perimeter of the nested element is much more critical. Interestingly, the additional freedom of the semi-circular nested elements allows optimizing them for a better loss performance than the ideal full-circle design.
\end{abstract}

Index Terms-Microstructured fibers; Fiber optics; Fiber properties; Fiber design and fabrication.

\section{INTRODUCTION}

$\mathrm{H}$ OLLOW-core fibers [1] have been studied and developed over the past decades due to their remarkable properties and several potential applications. These fibers can be used in applications such as high power delivery [2], ultra-short pulse delivery [3], pulse compression [4], mid-infrared (mid-IR) transmission [5], and terahertz applications [6]. A key feature of the hollow-core fiber is that most of the light can be guided inside the air-core and the optical damage threshold is raised while the material absorption is reduced. Two types of guiding mechanisms have been identified to explain the optical properties of hollow-core fibers in the last decade. The first one guides light inside the air-core via the photonic bandgap effect, while the second type guides light via inhibited coupling between the core-guided modes and the continuum of modes of the cladding or von Neumann-Wigner bound states in a continuum [7], including also the so-called hollowcore anti-resonant (HC-AR) fibers guiding through the antiresonant effect. The inhibited coupling mechanism was reported by Couny et al. [7] in which the inhibited coupling

Manuscript received June 1, 2015. (Write the date on which you submitted your paper for review.)

The authors are with the DTU Fotonik, Department of Photonics Engineering, Technical University of Denmark, DK-2800, Kgs. Lyngby, Denmark (e-mail: seha@fotonik.dtu.dk, oban@fotonik.dtu.dk, and moba@fotonik.dtu.dk, phone: +45 4523775, fax: +45 45936581). between the core-guided modes and cladding modes is explained by the high degree of the transverse-filed mismatch between the core and cladding modes [7]. These fibers offer much broader spectral bandwidths with low losses. Recently, HC-AR fibers with "negative-curvature" core boundaries have been proposed and designed by several research groups as an alternative optical transmission medium owing to the low transmission loss and broad transmission bands [8]-[17]. The coupling inhabitation between the core and cladding modes can be enhanced by using negative curvature (or hypocycloid) core contour [8], [9]. These fibers also offer weak overlap of the core modes with the silica part of the fiber [7]-[9], [17], which is one of the essential requirements for high power beam delivery.

A simple negative-curvature HC-AR fiber design was proposed and fabricated in [18] where eight contiguous circular capillary tubes used in the cladding acting as antiresonant elements that define the negative-curvature core. Light transmission above $3.5 \mu \mathrm{m}$ was reported in [10]; despite the very high material loss of silica in this spectral regime. The importance of the negative-curvature on transmission loss was also discussed in [10], and subsequently it was also pointed out that a "node-free" (i.e. where the cladding tubes do not touch each other) core boundary seems critical as well [14].

A modified form of HC-AR fiber was proposed in [13], where extra anti-resonant smaller glass tubes are nested inside the original glass tubes which defines the core. The leakage loss is reduced owing to the weak overlap between the core and cladding modes. Multiple nested elements can reduce the loss even further [15]; whether these additional anti-resonant elements be nested inside the first nested elements [15] or be adjacent to each other [17], such designs offer extra degree of freedom needed for increasing the extinction loss ratio of the fundamental mode to the higher-order modes in the both nearIR and mid-IR spectral regimes.

The aim of this work is to understand the role of the nested element in the overall loss performance of the HC-AR fiber, in particular concerning the position and curvature of the nested elements. It is clear that nested elements in general tend to increase the screening of the core modes so that the leakage loss is reduced, and that one can also find compromises where the higher-order modes are screened to a lesser degree than the fundamental mode, thus increasing the so-called higher-ordermode extinction ratio, which is critical for effective singlemode operation. Until now, though, only a full circular nested 
element has been considered, but the question is how the core mode is influenced if the nested element interior boundary position is kept fixed while its curvature is changed, or when the nested element interior boundary curvature is fixed and its position is changed. Such "semi-circular" scenarios could come up in a production stage, and even if they seem less ideal than a nested element consisting of a perfect full circle, we here show that often the penalty is quite small and in some cases the semi-circle design might even improve the loss performance of the fiber. That notwithstanding, the main goal of the paper is to get an intuition about whether it is the curvature or the position of the nested element that is critical for the optimal low-loss performance, and our results point towards the position as the most critical parameter.

\section{NUMERICAL ANALYSIS}

We used the finite-element method based commercial software COMSOL for our numerical simulations. Perfectlymatched layer boundary conditions were used to accurately calculate the leakage loss. In order to model HC-AR fibers accurately, both mesh size and perfectly-matched layer parameters must be optimized (see also [13],[17] for details).

As the structure reported in [17] for near-IR calculations, we consider an HC-AR fiber with 6 circular anti-resonant tubes, fixing the core radius to $R_{\mathrm{c}}=15.67 \mu \mathrm{m}$ (defined as the maximum radius of a circle that can be inscribed inside the core). We have chosen a silica strut thickness of $t=0.42 \mu \mathrm{m}$ so that an anti-resonant first-order transmission window has a loss minimum in the near-IR range (specifically around $\lambda=1.06 \mu \mathrm{m}$, the suitably chosen target wavelength of our designs). Figure 1 shows the three considered designs, all shown in the configuration optimized to give minimal leakage loss at $\lambda=1.06 \mu \mathrm{m}$. Figure 1(a) shows the geometry of a "typical" HC-AR fiber [16] with six anti-resonant tubes in which tubes are separated each other forming so-called nodefree configuration. The modified HC-AR fiber proposed by Poletti [15], is shown in Fig. 1(b) in which smaller tubes having diameter of $d_{\mathrm{i}}$ are nested with the original tubes of diameter $d_{0}$. It is here shown in the configuration giving the lowest leakage loss at $1.06 \mu \mathrm{m}$ [17]. The structure under investigation is shown in Fig. 1(c), using semi-circular antiresonant tubes. The perimeter distance between the outer tubes is $1.33 \mu \mathrm{m}$ for all designs. The separation between the inner and outer tubes along the radial direction is indicated by $\mathrm{z}$ which is $11.17 \mu \mathrm{m}$ for the minimal leakage loss shown in Fig. $1(\mathrm{~b}-\mathrm{c})$.

First, we investigated the effect of changing the inner airhole radius $r_{1}$ while keeping the distance between the core boundary and the interior boundary of the nested element, $z$, fixed to $11.17 \mu \mathrm{m}$ (this is the value that comes out when optimizing the $1 \mathrm{AE}$ structure to give the lowest leakage loss). We specifically monitor the leakage loss and higher-ordermode extinction ratio (HOMER). The HOMER is defined as the ratio between the transmission loss of the HOM having the lowest transmission loss and the transmission loss of the fundamental mode (FM), which determines the effectively single-mode operation of a fiber. The change in the inner airhole radius allows us to investigate several fiber structures

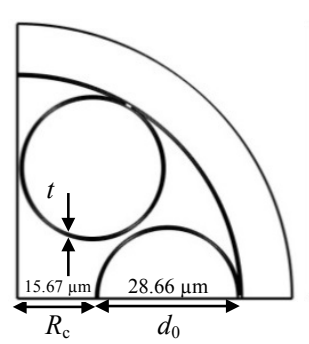

(a)

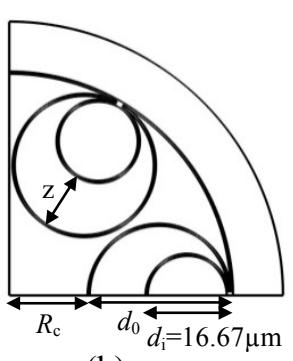

(b)

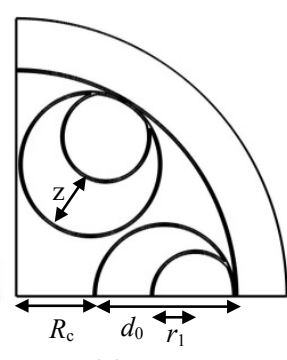

(c)
Fig. 1. Geometries considered in the near-IR simulations. (a) Typical optimized HC-AR fiber design with six circular tubes; (b) the nested ("1AE", i.e. one anti-resonant element) design proposed in [15] in which $d_{\mathrm{i}}=16.67 \mu \mathrm{m}$; (c) our proposed structure with one semi-circular anti-resonant element (1SAE). All fibers have the same core radius $R_{\mathrm{c}}=15.67 \mu \mathrm{m}, d_{\mathrm{o}}=28.66 \mu \mathrm{m}$ and uniform silica strut thickness $t=0.42 \mu \mathrm{m}$.

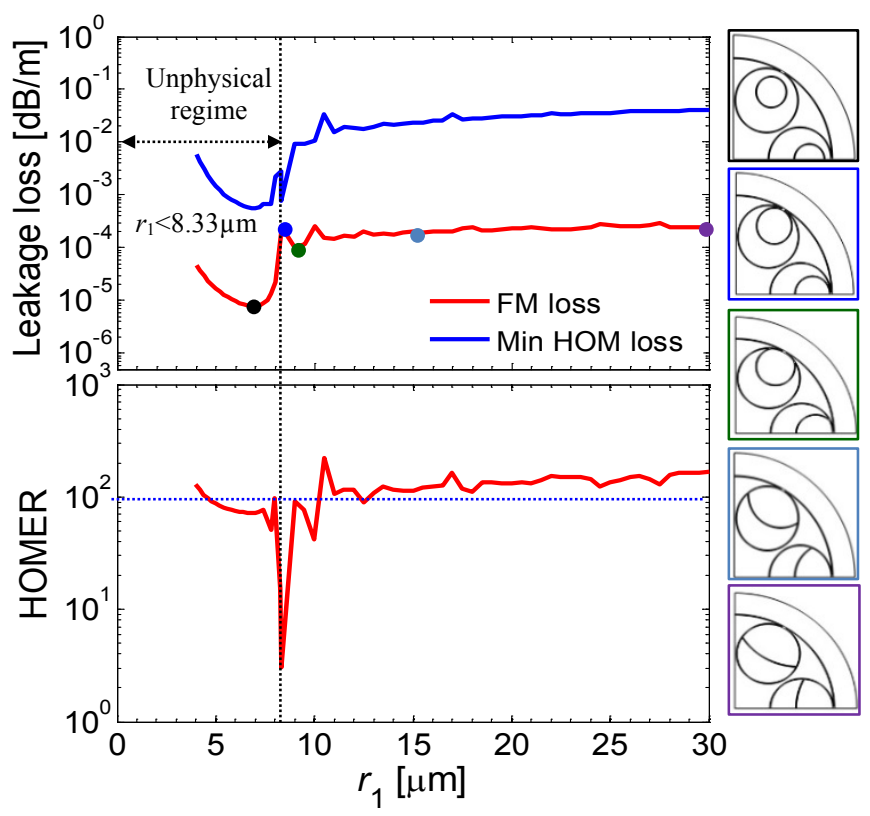

Fig. 2. Calculated leakage loss and HOMER as a function of the inner air-hole radius $r_{1}$ keeping $z$ fixed to $11.17 \mu \mathrm{m}$. All structures have the same core radius $R_{\mathrm{c}}=15.67 \mu \mathrm{m}$ and uniform silica strut thickness $t=0.42 \mu \mathrm{m}$. The color of the frame corresponds to the color of the dot points in the plot.

with the same core radius $R_{\mathrm{c}}$, silica strut thickness $t$, and the separation between the inner and outer tubes $z$; the only thing that changes is that as the nested element becomes semicircular, its curvature becomes more and more flat. The geometrical configurations for different inner air-hole radii are shown in the right hand side of Fig. 2. The inner air-hole radius $r_{1}=8.33 \mu \mathrm{m}$ corresponds to the original $1 \mathrm{AE}$ structure proposed in [15], so when $r_{1}<8.33 \mu \mathrm{m}$ the inner tube will be "floating" in the outer tube (an unphysical scenario, but nevertheless possible to model), and when $r_{1}>8.33 \mu \mathrm{m}$ the inner air-hole tube will no longer be a full circle and instead becomes semi-circular. It can be seen from Fig. 2 that a dramatic change in the leakage loss is observed when the inner and outer tubes start floating at around $r_{1}=7 \mu \mathrm{m}$ while it is only weakly changing in between $r_{1}=8.33-30 \mu \mathrm{m}$. The minimum leakage loss (that is for physical values of the radius) is actually obtained at $r_{1}=9 \mu \mathrm{m}$ when the inner air-hole tubes start being semi-circularly shaped. Thus, we conclude that the curvature plays a minor role as there is only around a 


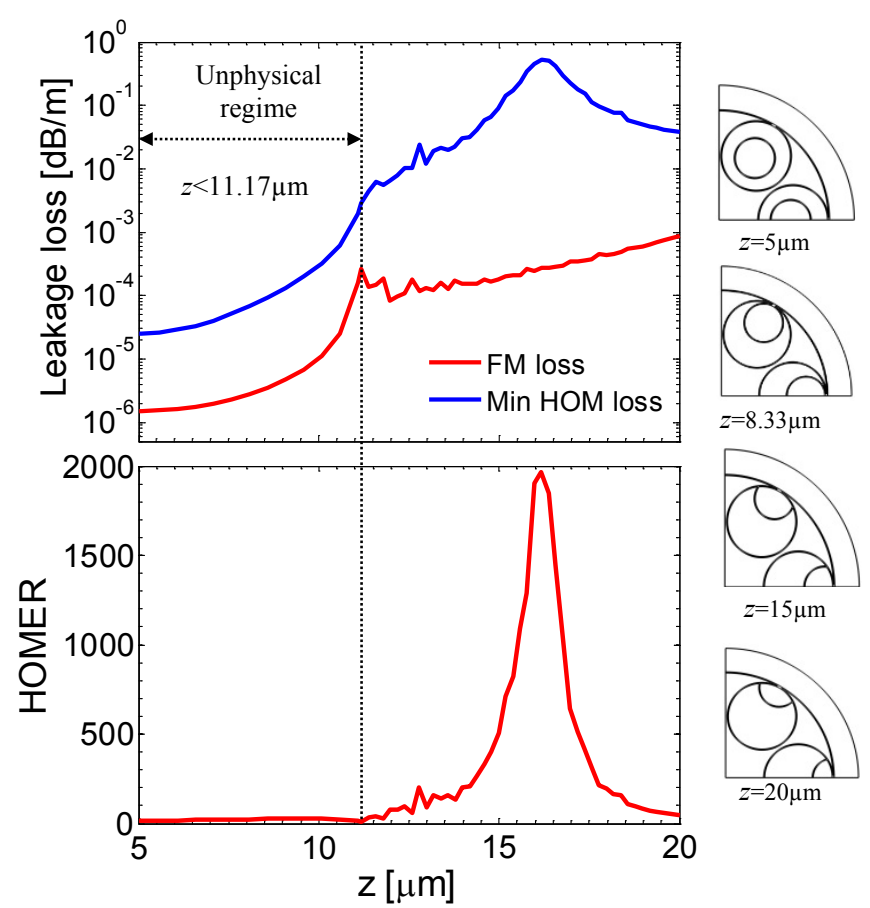

Fig. 3. Calculated leakage loss and HOMER as a function of the tube separation $z$ keeping $r_{1}$ fixed to $8.33 \mu \mathrm{m}$. All structures have the same core radius $R_{\mathrm{c}}=15.67 \mu \mathrm{m}$ and uniform silica strut thickness $t=0.42 \mu \mathrm{m}$.

factor of 2 variations from the full circle to an almost flat line. In other words, the penalty for having an imperfect nested element or even just a "crossbar" in the outer circle is insignificant. Important to note, the small ripples (fluctuations) of FM and minimum HOM loss is due to the anti-crossings with ring guided modes of high azimuthal number [15], [19]. Given this trend it is not evident that the dramatic drop in loss as the circle starts to float (the unphysical case) is related to the increased negative curvature as much as the fact that the nested element no longer touches the outer tube. The HOMER is plotted in the lower frame, and interestingly, we found lowest HOMER for the original $1 \mathrm{AE}$ design. The HOMER can be made higher by reshaping the size of the inner antiresonant tubes and we found HOMER to reach a value of more than 100 when $r_{1}$ is higher than $13 \mu \mathrm{m}$. That being said, also HOMER is quite constant as the nested element curvature flattens. Second, the effect of changing the tube separation $z$ while keeping $r_{1}$ fixed (to th

e value from the loss-optimized $1 \mathrm{AE}$ design) is investigated which is shown in Fig. 3. A dramatic drop in leakage loss is also observed for the unphysical case. The maximum HOMER is found to be $\sim 2000(z \sim 16.20 \mu \mathrm{m})$ which is far higher than the original $1 \mathrm{AE}$ design $(z=11.17 \mu \mathrm{m})$. We observe that the HOMER value increases as the nested tube is removed away from the core boundary; while the FM leakage loss is almost constant from the starting value and the HOM loss increases quickly. The leakage loss starts increasing when $\mathrm{z}>16.20 \mu \mathrm{m}$ and the change in the leakage loss is much more pronounced by the variation of tube separation compared to the variation of $r_{1}$ (see Fig. 2). We conclude that the FM leakage loss increases somewhat when the distance is increased, but since the HOM losses increase much faster the HOMER quickly becomes extremely large. Therefore the distance has a much more pronounced effect than the curvature on the loss performance. To understand the coupling effect between the core-guided modes and cladding modes we have plotted the effective mode index $\left(\mathrm{n}_{\mathrm{eff}}\right)$ and leakage loss as a function of $z$ from 12 to $20 \mu \mathrm{m}$ which is shown in Fig. 4. It can be seen from the effective index profile that the effective index of the FM core- mode $\left(\mathrm{LP}_{01}\right)$ has higher refractive index than the cladding modes which results in weak coupling between the core and cladding modes and remains almost independent of $z$. The core-guided HOM experiences a strong phase matching with the cladding modes at $\mathrm{z} \sim 16.20 \mu \mathrm{m}$ which results in high HOM loss. A weak interaction between the core-guided HOMs and cladding modes take place away from the phase matching (see modal spectrum for $z=12 \mu \mathrm{m}$ and for $z=20 \mu \mathrm{m}$ ) [20].

Next we investigate the $1 \mathrm{AE}$ design in the case where it is optimized to give the maximum HOMER value; as mentioned in [17] the design needs to sacrifice the lowest loss in order to maximize HOMER. First we change the inner air-hole radius $r_{1}$ while keeping the separation $z$ fixed to the value $15.83 \mu \mathrm{m}$ found in the $1 \mathrm{AE}$ optimized design. It can be seen from Fig. 5 that leakage loss is almost constant by the variation of inner air-hole radius; this is similar trend to what we found for the optimized loss case (see Fig. 2). However, in this case the HOM losses decrease when the nested curvature flattens, so the HOMER decreases with the increase of the inner air-hole radius.

Lastly, the effect of tube separation $\mathrm{z}$ on the leakage loss and HOMER is also investigated. It can be seen from Fig. 6 that the leakage loss increases with the increase of the distance between the core and the interior boundary of the nested tube $z$, while keeping the curvature fixed. The leakage loss dramatically decreases for the unphysical case when $z<15.83$ $\mu \mathrm{m}$. Therefore, we can say that the tube separation $z$ plays a more important role on the leakage loss compared to the curvature of the nested tubes (when compared to Fig. 2 and 5). Notably, maximum HOMER is obtained for the original $1 \mathrm{AE}$ design i.e., $z \sim 16 \mu \mathrm{m}$ and $r_{1}=6 \mu \mathrm{m}$.
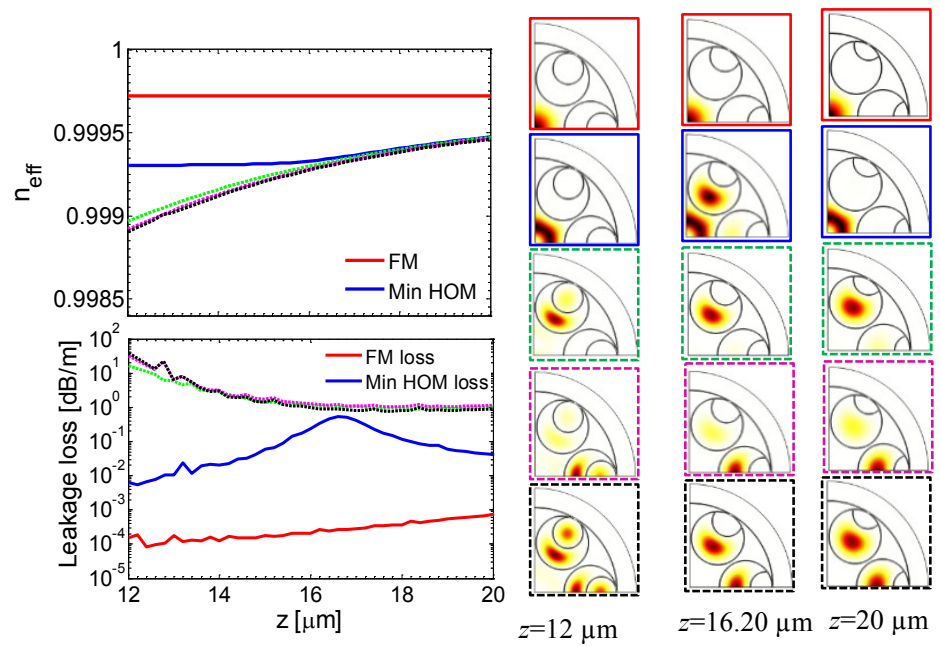

Fig. 4. Effect of changing tube separation $\mathrm{z}$ on effective mode index and leakage loss. All structures have the same core radius $R_{\mathrm{c}}=15.67 \mu \mathrm{m}, \mathrm{r}_{1}=8.33$ 
$\mu \mathrm{m}$, and uniform silica strut thickness $t=0.42 \mu \mathrm{m}$. The color of the frame corresponds to the color of the line in the plot.

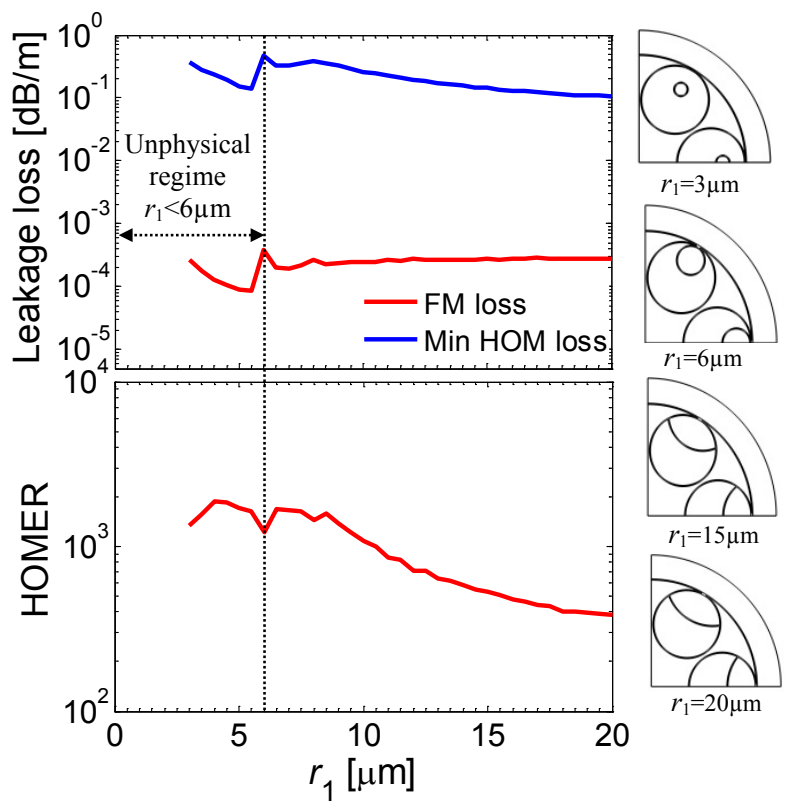

Fig. 5. Effect of changing $r_{1}$ on leakage loss and HOMER with a fixed $R_{\mathrm{c}}=15.67 \mu \mathrm{m}, t=0.42 \mu \mathrm{m}, z=15.83 \mu \mathrm{m}, d_{\mathrm{o}}=28.66 \mu \mathrm{m}$ and $\lambda=1.06 \mu \mathrm{m}$.
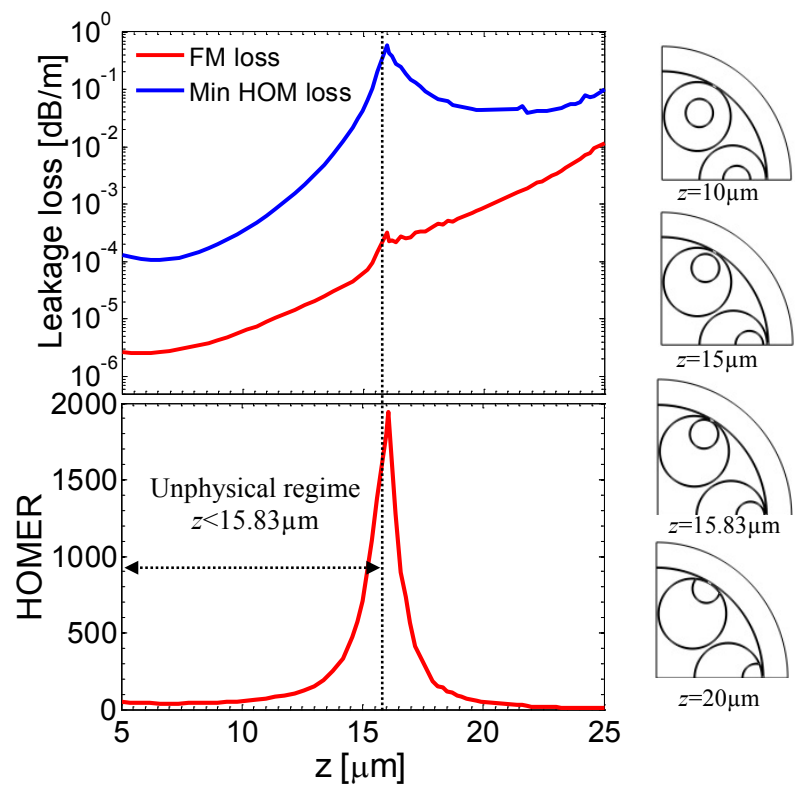

Fig. 6. Effect of changing $z$ on leakage loss and HOMER with a fixed $R_{\mathrm{c}}=15.67 \mu \mathrm{m}, t=0.42 \mu \mathrm{m}, r_{1}=6 \mu \mathrm{m}, d_{\mathrm{o}}=28.66 \mu \mathrm{m}$ and $\lambda=1.06 \mu \mathrm{m}$.

The effect of changing $r_{1}$ and $z$ on the leakage loss is shown in Fig. 7. It can be seen from Fig. 7 that the leakage loss is almost constant in between $z=11.17$ to $18 \mu \mathrm{m}$ and $r_{1}=9$ to $17 \mu \mathrm{m}$. The loss is quite constant along $r_{1}$ for a given $z$, while generally the loss increases as $z$ is increased for a fixed $r_{1}$. This supports the conclusion found above that the curvature plays a minor role, while the distance from

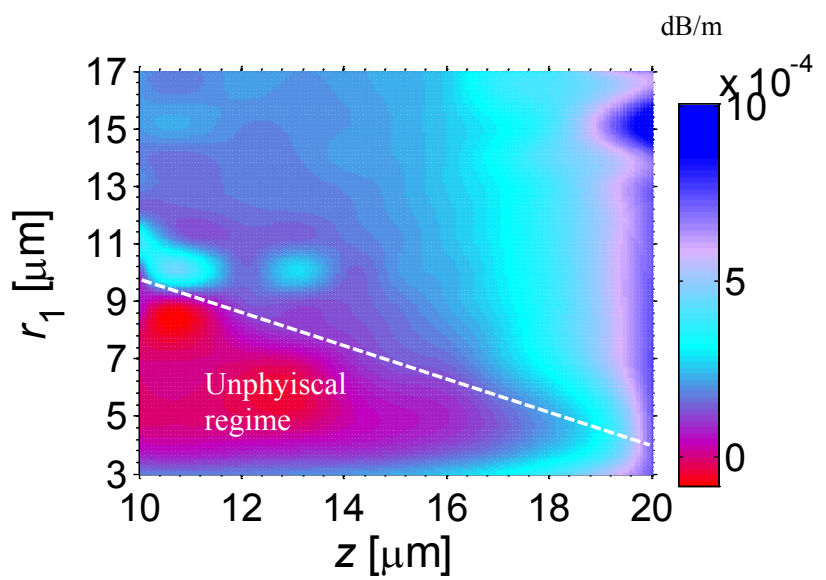

the core to the interior boundary position of the nested element is more critical.

Fig. 7. Calculated leakage loss with different values of $r_{1}$ as a function of $z$. The simulations are performed at $\lambda=1.06 \mu \mathrm{m}$.

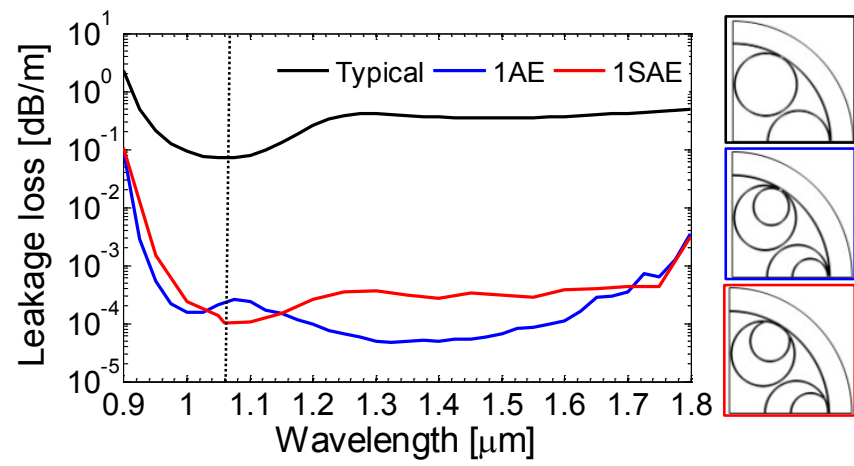

Fig. 8. Calculated loss spectra. All fibers have the same core radius $R_{\mathrm{c}}=15.67 \mu \mathrm{m}, d_{\mathrm{o}}=28.66 \mu \mathrm{m}$ and uniform silica strut thickness $t=0.42 \mu \mathrm{m}$. The optimum parameters for $1 \mathrm{AE}$ and $1 \mathrm{SAE}$ structures are $z=11.17 \mu \mathrm{m}$, $r_{1}=8.33 \mu \mathrm{m}$ and $z=11.17 \mu \mathrm{m}, r_{1}=9 \mu \mathrm{m}$ respectively. The color of the frame corresponds to the color of the line in the plot. The thin dotted line indicates the wavelength for which the losses are optimized.

The leakage loss for the optimized parameters as a function of wavelength in the near-IR spectral regime is shown in Fig 8. We have neglected the material loss of silica to calculate the total loss because the measured loss of dry F300 silica is of the order of $10^{-3} \mathrm{~dB} / \mathrm{m}$ [13] or less in the wavelength range between 0.95 and $1.40 \mu \mathrm{m}$. The calculated power fraction in silica of the optimum $1 \mathrm{SAE}$ structure with the lowest leakage loss is less than $10^{-4}$ in this spectral regime, and similar values are found for the 1AE structure. Therefore, the transmission loss will practically be equivalent to the leakage loss. It can be seen from the leakage loss spectra of Fig. 8 that the 1SAE fiber has a leakage loss of $0.1 \mathrm{~dB} / \mathrm{km}$ at $1.06 \mu \mathrm{m}$, which is roughly a factor of 2 lower than $1 \mathrm{AE}$. This shows that at the target wavelength, the semi-circular design 1SAE actually outperforms the ideal full-circle design 1AE, although over the whole spectrum the $1 \mathrm{AE}$ design performs 


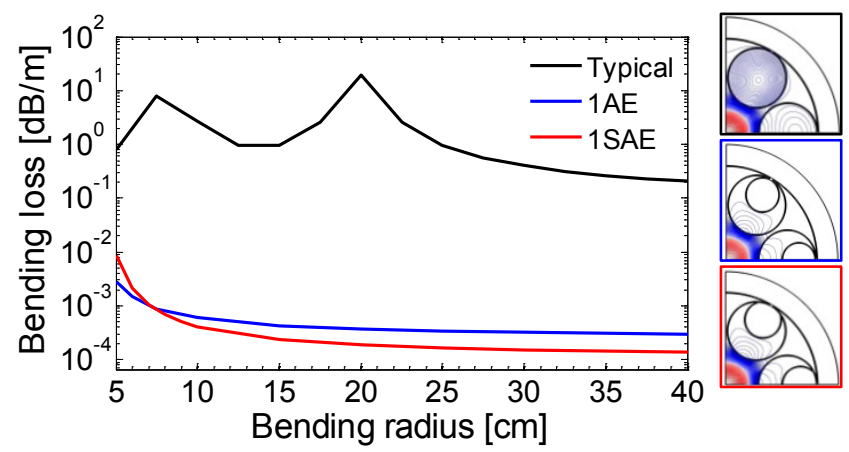

Fig. 9. Calculated bending loss at $1.06 \mu \mathrm{m}$ versus bending radius; all chosen designs are optimized to give the lowest leakage loss. The contour plots of the fundamental air-core mode distribution are shown in the right hand side for a $10 \mathrm{~cm}$ bending radius. The color of the frame corresponds to the color of the line in the plot.
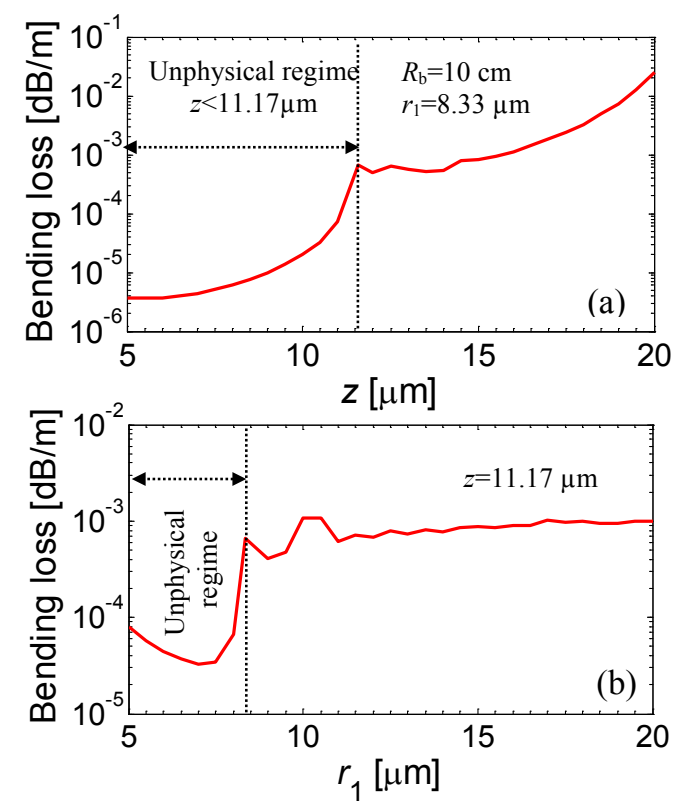

Fig. 10. Calculated bending loss as a function of (a) tube separation $z$ (b) inner air-hole radius $r_{1}$ for $10 \mathrm{~cm}$ bending radius at $1.06 \mu \mathrm{m}$.
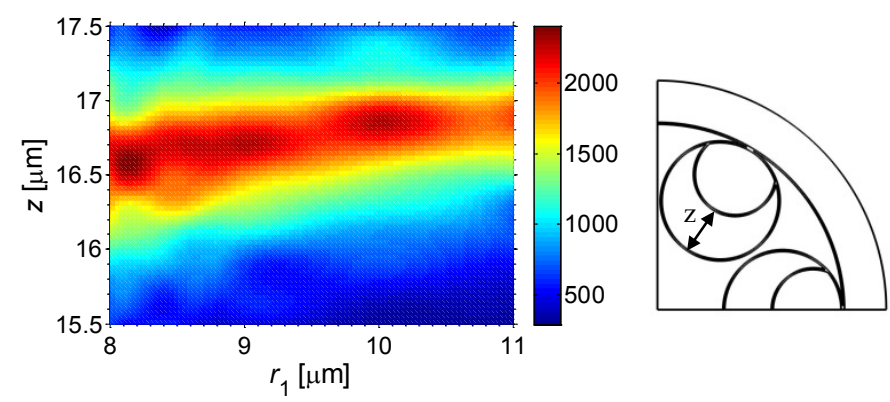

Fig. 11. Calculated HOMER with different values of $z$ as a function of $r_{1}$. The simulations are performed at $\lambda=1.06 \mu \mathrm{m}$.

slightly better. It is remarkable to see that the lowest leakage loss for the $1 \mathrm{AE}$ design is found at a longer wavelength than the $\lambda=1.06 \mu \mathrm{m}$ wavelength it is optimized for, unlike the "typical" and 1SAE designs. This can happen because the optimization is done only locally.

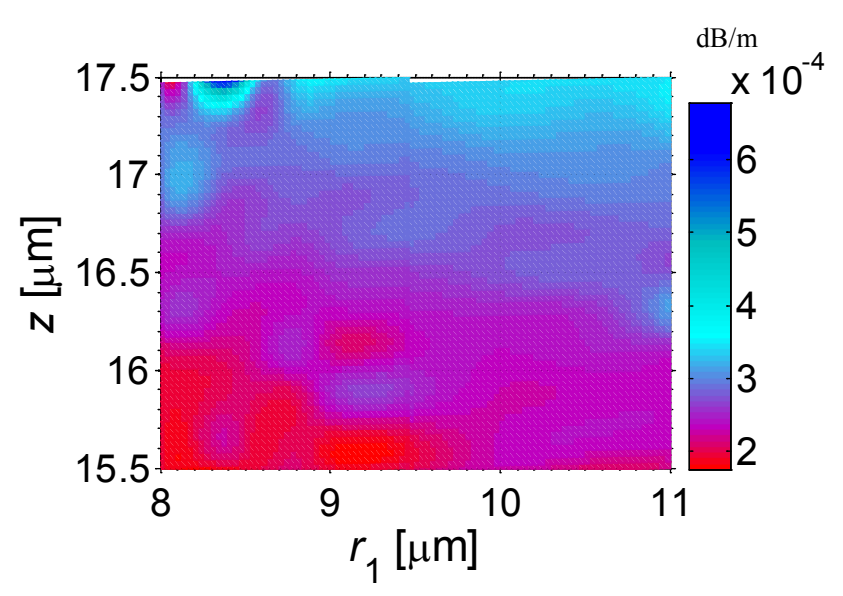

Fig. 12. Calculated leakage loss with different values of $z$ as a function of $r_{1}$. The simulations are performed at $\lambda=1.06 \mu \mathrm{m}$.

Let us now consider the bending loss, which is calculated by transforming the bent structure into its equivalent straight structure with equivalent refractive index profile, $n_{\text {eq }}$ defined by [21]

$$
n_{\mathrm{eq}}=n(x, y) \exp \left(x / R_{\mathrm{b}}\right)
$$

where $R_{\mathrm{b}}$ is the bending radius, $x$ is the transverse distance from the center of the fiber, $n(x, y)$ is the refractive index profile of the straight fiber. The bending loss was calculated at $1.06 \mu \mathrm{m}$ and the bending direction was chosen along the $\mathrm{x}$-axis giving the bend-loss curve shown in Fig. 9. Evidently the 1SAE design has a lower bending loss than the $1 \mathrm{AE}$ design for bend radii between 7 and $40 \mathrm{~cm}$ which is due to the weak coupling between the core-guided mode and the cladding modes [14] and the leakage loss is only weakly changing for bend radii above $\sim 20 \mathrm{~cm}$, but for low bending radius the $1 \mathrm{AE}$ design shows lower bending loss than 1SAE design. It can also be seen from Fig. 9 that a few loss peaks were observed for the "typical" design as the bend radius is decreased, while no loss peaks were observed for the 1AE and 1SAE designs.

The bending loss as a function of the tube separation $z$ $\left(r_{1}=8.33 \mu \mathrm{m}\right)$ and inner air-hole radius $r_{1}(z=11.17 \mu \mathrm{m})$ is shown in Fig. 10. The bending loss increases sharply for a tube separation larger than $15 \mu \mathrm{m}$ whereas the bending loss is weakly changing by the variation of the inner air-hole radius $r_{1}$. Again we may conclude that changing the nested element curvature gives an insignificant penalty in the performance, while the distance is much more critical.

The semi-circular nested element design offers an extra degree of design freedom compared to the full-circle nested element design. We therefore need to perform a 2D optimization of HOMER, which is shown in Fig. 11. It is performed by systematically changing the separation between the inner and outer air-hole tubes $z$ and inner airhole radius $r_{1}$. The optimum HOMER is found to be over 2,000 at $z=16.5 \mu \mathrm{m}$ and $r_{1}=8.2 \mu \mathrm{m}$. The corresponding leakage loss of the FM is shown in Fig. 12 in which we see that the leakage loss is weakly changing by the variation of 
both $z$ and $r_{1}$, but clearly the design with the lowest leakage loss is not the same as the design with the highest HOMER.

For completeness Fig. 13 shows the wavelength dependence of the leakage loss of the FM, the minimum HOM loss, and the HOMER in the near-IR spectral regime; for each design the structure is optimized to give the maximal HOMER. HOMER can be made in excess of 1,000 in the range of 1.1-1.15 $\mu \mathrm{m}$ and over 2,000 at 1.06 $\mu \mathrm{m}$ for $r_{1}=8.2 \mu \mathrm{m}$, and $z=16.5 \mu \mathrm{m}$, which makes the fiber effectively single-moded in the near-IR spectral regime. Also the $1 \mathrm{AE}$ design has excellent HOMER performance, and at the same time it has a lower leakage loss than $1 \mathrm{SAE}$ design.
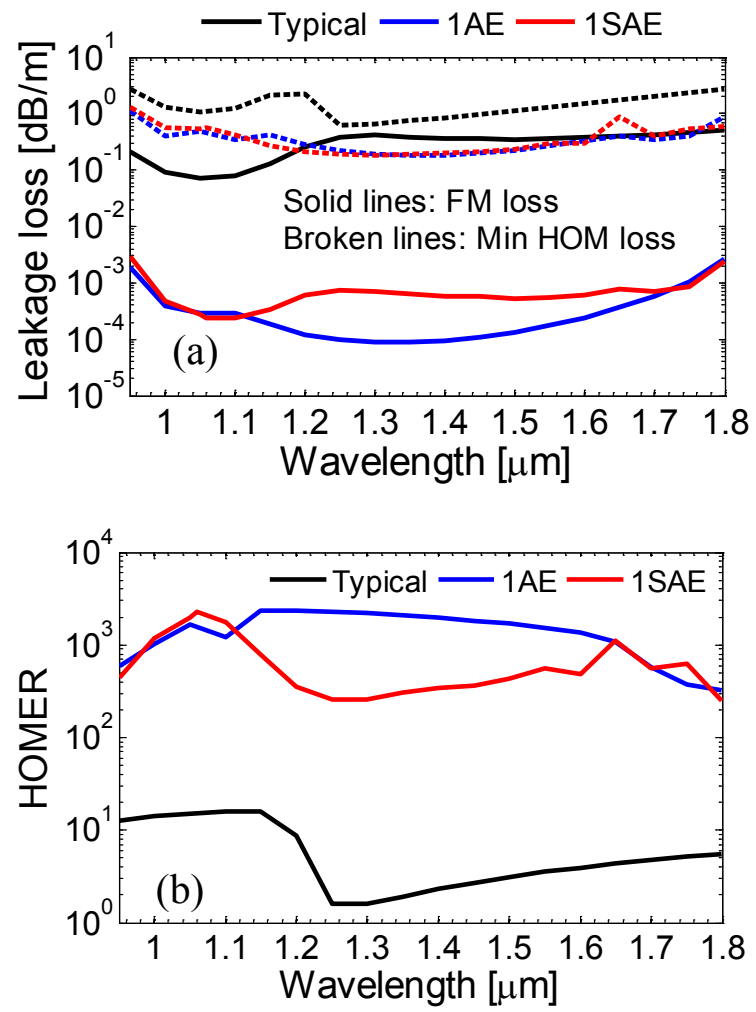

Fig. 13. Wavelength dependence of (a) leakage loss and (b) HOMER for the optimized parameters. The optimum design parameters are: $R_{\mathrm{c}}=15.67$ $\mu \mathrm{m}, t=0.42 \mu \mathrm{m}, d_{0}=28.66 \mu \mathrm{m}$, and inner air-hole radius $r_{1}=6 \mu \mathrm{m}$, and $8.2 \mu \mathrm{m}$ for $1 \mathrm{AE}$, and $1 \mathrm{SAE}$ respectively.

\section{CONCLUSION}

Concluding, we have thoroughly investigated the effect of the shape and position of a single nested anti-resonant element on the overall loss performance of a hollow-core anti-resonant fiber. We allowed the circular nested element to become semi-circular, and thus we could independently investigate the effects of curvature and position of the nested element. We found through numerical simulations that changing the distance between the core boundary and the nested anti-resonant element boundary significantly affects the loss performance; in some cases it deteriorates the performance while in other cases is vastly improves the performance. We even found that the semi-circular case could outperform the optimized full-circle nested element design. Instead the curvature of the nested element had a very little impact on the loss performance. This means that it is not crucial to have a nested element with a negative curvature, but its position is much more crucial. These results should give a better intuition when designing antiresonant fibers with nested elements, and we expect that this will lead to novel designs with improved performance.

\section{ACKNOWLEDGMENT}

The authors acknowledge fruitful discussions with $\mathrm{F}$. Poletti, W. Belardi, and J. Ju.

\section{REFERENCES}

[1] R. F. Cregan, R. F. Cregan, B. J. Mangan, J. C. Knight, T. A. Birks, P. S. J. Russel, P. J. Roberts, and D. C. Allan, "Singlemode photonic band gap guidance of light in air," Science, vol. 285, no. 5433, pp. 1537-1539, Sep. 1999.

[2] G. Humbert, J. C. Knight, G. Bouwmans, P. S. J. Russell, D. Williams, P. Roberts, and B. Mangan, "Hollow core photonic crystal fibers for beam delivery," Opt. Express, vol. 12, no. 8, pp. 1477-1484, Apr. 2004.

[3] F. Gèrôme, P. Dupriez, J. Clowes, J. C. Knight, and W. J. Wadsworth, "High power tunable femtosecond soliton source using hollow-core photonic bandgap fiber, and its use for frequency doubling," Opt. Express, vol. 16, no. 4, pp. 23812386, Feb. 2008.

[4] F. Gérôme, K. Cook, A. K. George, W. J. Wadsworth, and J. C. Knight, "Delivery of sub-100fs pulses through $8 \mathrm{~m}$ of hollowcore fiber using soliton compression," Opt. Express, vol. 15, no. 12, pp. 7126-7131, Jun. 2007.

[5] A. Urich, R. R. J. Maier, F. Yu, J. C. Knight, D. P. Hand, and J. D. Shephard, "Flexible delivery of Er:YAG radiation at $2.94 \mu \mathrm{m}$ with negative curvature silica glass fibers: a new solution for minimally invasive surgical procedures," Biomed. Opt. Express, vol. 4, no. 2, pp. 193-205, Feb. 2013.

[6] J. Anthony, R. Leonhardt, S. G. Leon-Saval, and A. Argyros, "THz propagation in kagome hollow-core microstructured fibers," Opt. Express, vol. 19, no. 19, pp. 18470-18478, Sep. 2011.

[7] F. Couny, F. Benabid, P. J. Roberts, P. S. Light, and M. G. Raymer, "Generation and photonic guidance of multi-octave optical-frequency combs," Science, vol. 318, no. 5853, pp. 1118-1121, Nov. 2007.

[8] Y. Y. Wang, F. Couny, P. J. Roberts, and F. Benabid, "Low loss broadband transmission in optimized core-shape Kagome hollow-core PCF," Lasers Electro-Optics Quantum Electron. Laser Sci. Conf. (QELS), 2010 Conf., pp. 4-5, 2010.

[9] Y. Y. Wang, N. V Wheeler, F. Couny, P. J. Roberts, and F. Benabid, "Low loss broadband transmission in hypocycloid-core Kagome hollow-core photonic crystal fiber," Opt. Lett., vol. 36, no. 5, pp. 669-671, 2011.

[10] F. Yu, W. J. Wadsworth, and J. C. Knight, "Low loss silica hollow core fibers for 3-4 $\mu \mathrm{m}$ spectral region," Opt. Express, vol. 20, no. 10, pp. 11153-11158, May 2012.

[11] W. Belardi and J. C. Knight, "Effect of core boundary curvature on the confinement losses of hollow antiresonant fibers," Opt. Express, vol. 21, no. 19, pp. 21912-21917, 2013.

[12] A. N. Kolyadin, A. F. Kosolapov, A. D. Pryamikov, A. S. Biriukov, V. G. Plotnichenko, and E. M. Dianov, "Light transmission in negative curvature hollow core fiber in extremely high material loss region," Opt. Express, vol. 21, no. 8, pp. 9514-9519, 2013.

[13] W. Belardi and J. C. Knight, "Hollow antiresonant fibers with reduced attenuation," Opt. Lett., vol. 39, no. 7, pp. 1853-1856, Apr. 2014.

[14] W. Belardi and J. C. Knight, "Hollow antiresonant fibers with low bending loss," Opt. Express, vol. 22, no. 8, pp. 9514-9519, 2014.

[15] F. Poletti, "Nested antiresonant nodeless hollow core fiber," Opt. Express, vol. 22, no. 20, pp. 23807-23828, Sep. 2014. 
[16] A. D. Pryamikov, A. S. Biriukov, A. F. Kosolapov, V. G. Plotnichenko, S. L. Semjonov, and E. M. Dianov, "Demonstration of a waveguide regime for a silica hollow-core microstructured optical fiber with a negative curvature of the core boundary in the spectral region $>3.5 \mu \mathrm{m}$," Opt. Express, vol. 19, no. 2, pp. 1441-1148, Jan. 2011.

[17] M. S. Habib, O. Bang, and M. Bache, "Low-loss hollow-core silica fibers with adjacent nested anti-resonant tubes," Opt. Express, vol. 23, no. 13, pp. 17394-17406, 2015.

[18] A. F. Kosolapov, A. D. Pryamikov, A. S. Biriukov, V. S. Shiryaev, M. S. Astapovich, G. E. Snopatin, V. G. Plotnichenko, M. F. Churbanov, and E. M. Dianov, "Demonstration of $\mathrm{CO}_{2}$ laser power delivery through chalcogenide-glass fiber with negative-curvature hollow core," Opt. Express, vol. 19, no. 25, pp. 25723-25728, Dec. 2011.

[19] L. Vincetti and V. Setti, "Waveguiding mechanism in tube lattice fibers," Opt. Express, vol. 18, no. 22, pp. 23133-23146, 2010.

[20] M. C. Günendi, P. Uebel, M. H. Frosz, and P. S. J. Russell, "Broad-band robustly single-mode hollow-core PCF by resonant filtering of higher order modes," arXiv: 1508.06747, 2015.

[21] M. Heiblum and J. Harris, "Analysis of curved optical waveguides by conformal transformation," IEEE J. Quantum Electron., vol. 11, no. 2, pp. 75-83, 1975.

Md. Selim Habib (S'13) received the B.Sc. and M.Sc. Degrees in Electrical and Electronic Engineering from Rajshahi University of Engineering \& Technology, Rajshahi, Bangladesh in 2008 and 2012 respectively. He is currently pursuing the Ph.D. degree at DTU Fotonik, Department of Photonics Engineering, Technical University of Denmark, DK-2800, Kgs. Lyngby, Denmark.

Mr. Habib is a student member of the Optical Society of America, SPIE and the Danish Optical Society. He received the University Gold Medal Award from Rajshahi University of Engineering \& Technology in 2014.

Ole Bang received the M.S. degree in electrical engineering and the Doctor of Philosophy degree in nonlinear physics from the Technical University of Denmark (DTU) in 1991 and 1993, respectively. He was a Post-Doctoral Fellow with Laboratoire de Physique, Ecole Normale Supérieure de Lyon, France, from 1993 to 1995, where he worked on discrete physical models of the nonlinear dynamics of biomolecules. From 1995 to 1999, he was a Research Fellow with the Optical Sciences Centre, Australian National University, where he worked theoretically on nonlinear optics, in particular on solitons and modulational instability in materials with a quadratic nonlinearity and quasi-phase-matching gratings. In 1999 he became an Associate Professor at the Department of Informatics and Mathematical Modeling at DTU, continuing the work on nonlinear optics in quadratic nonlinear crystals. In 2003 he moved to the Department of Photonics Engineering, DTU Fotonik, where he in 2011 became Professor in Advanced Photonics Materials.

$\mathrm{He}$ is heading the Fiber Sensors \& Supercontinuum Group with currently 18 members working on fabricating Polymer Optical Fibers (POFs) and polymer Photonic Crystal Fibers (PCFs) and using them for sensing of biomolecules, strain, humidity, and temperature, in particular with Fiber Bragg gratings. Another main part of his work is on supercontinuum generation and nonlinear fiber-optics in silica and softglass PCFs, as well as its use in imaging and spectroscopy.

Dr. Bang is a Fellow of the Optical Society of America and the Danish Optical Society.
Morten Bache received his MSc and $\mathrm{PhD}$ from the Technical University of Denmark (DTU) in 1999 and 2002, respectively. After 3 years as a postdoc in University of Insubria (Italy), he returned to DTU to Dept. of Photonics Engineering, where currently he is an associate professor. His main research interest is nonlinear optical phenomena with ultrafast femtosecond pulses. In particular the study of excitations of temporal solitons in bulk media or optical waveguides and fibers that allow achieving efficient frequency conversion, pulse compression and supercontinuum generation. His main focus over the past decade is solitons generated in quadratic nonlinear crystals through cascaded (phasemismatched) frequency conversion processes. This gives access to a unique self-defocusing nonlinearity that has many advantages compared to traditional self-focusing four-wave mixing processes, in particular that it is inherently filament free. Recently he also worked on hollow-core gasfilled fibers for broadband handling, transmission and nonlinear interaction of near to mid-IR pulses.

Dr. Bache is a member of the Optical Society of America and the Danish Physical Society. 